Originalien

Unfallchirurg $2022 \cdot 125: 295-304$

https://doi.org/10.1007/s00113-021-01013-7

Angenommen: 22. April 2021

Online publiziert: 10. Juni 2021

๑ Der/die Autor(en) 2021

\section{Redaktion}

W. Mutschler, München

H. Polzer, München

B. Ockert, München

Bernhard Wilhelm Ullrich ${ }^{1,2} \cdot$ Merle Ottich $^{2} \cdot$ Aaron Lawson McLean ${ }^{3}$. Thomas Mendel ${ }^{1,2}$. Gunther Olaf Hofmann ${ }^{1,2}$. Philipp Schenk $k^{1,4}$

${ }^{1}$ Klinik für Unfall-, Hand- und Wiederherstellungschirurgie, Universitätsklinikum Jena, Jena, Deutschland

${ }^{2}$ Klinik für Unfall- und Wiederherstellungschirurgie, BG Klinikum Bergmannstrost Halle, Halle, Deutschland

${ }^{3}$ Klinik für Neurochirurgie, Universitätsklinikum Jena, Jena, Deutschland

${ }^{4}$ Koordinationsabteilung Wissenschaft, Forschung und Lehre, BG Klinikum Bergmannstrost Halle, Halle, Deutschland

\title{
Lokales Wirbelsäulenprofil nach operativer Behandlung thorakolumbaler und lumbaler Frakturen
}

\section{Einfluss von Repositionstechnik und Knochenqualität}

und lumbalen Wirbelsäulenverletzungen (Th10-L5) aus den Jahren 2001-2016 durchsucht. Eingeschlossen wurden Patienten, welche initial bisegmental dorsal offen instrumentiert wurden. Entsprechend dem im Erfassungszeitraum etablierten Therapiestandard erfolgte eine Verlaufsuntersuchung ca. 6 Wochen nach initialer dorsaler Stabilisierung vor ventraler Operation. Betrachtet werden nur die Daten vor ventraler Operation.

Im Bilddokumentationssystem IMPAX6 $^{\circledR}$ (Agfa HealthCare GmbH, Bonn, Germany) wurden die Datensätze dieser Patienten herausgesucht. Hierbei war Voraussetzung, dass es Röntgenund CT-Aufnahmen präoperativ, direkt postoperativ und Verlaufsdaten gab.

Erfasst wurde neben Alter und Geschlecht die verwendete Operationstechnik (AO-Schanz-Pin-Technik [AT] und „Kluger Technik“ [KT]). In einem interdisziplinären Dienstsystem aus $3 \mathrm{Kli}$ niken (neurochirurgisch, orthopädisch und unfallchirurgisch) waren Operateure beider Schulen (AT und KT) an der Akutversorgung von Wirbelsäulenverletzungen beteiligt. Alle Operateure waren Oberärzte und Fachärzte. Die Knochenqualität wurde mittels $\mathrm{HU}$ gemessen, die Frakturmorphologie nach AOSpine klas- sifiziert und der bisegmentale GrundDeckplatten-Winkel (bGDW) auf Höhe der Verletzung gemessen [5].

Die AO-Schanz-Pin-Techniksteht seit Mitte der 1980er-Jahre zur Verfügung [6] . Diese nutzt den einliegenden Stab zur Reposition. Aktuell ist die Anwendung dieses Fixateur-Systems in den „surgical references" der AOSpine beschrieben [2]. Hierbei wird nach Einbringen der Pins der kraniokaudale Abstand der Pins dorsal auf dem bereits eingebrachten Stab fixiert und dann eine Lordosierung und Distraktion durchgeführt (AT), • Abb. 1. Ungefähr zeitgleich wurde die „KlugerTechnik" beschrieben, welche mit einem temporär zu montierenden Repositeur arbeitet [3]. Mit diesem wird über Verlängerungen der Pedikelschrauben beim Lordosieren eine kontrollierte Verkürzung der kraniokaudalen Distanz der Pedikelschraubenköpfe durchgeführt, was eine stärkere Lordosierung ermöglichen soll (KT). Die Distraktion wird nach Fixation der lordotischen Stellung durchgeführt (krypton ${ }^{\circledR}$ [Ulrich, Ulm, Germany] Repositionstechnik WS 2801 Rev01/1106), • Abb. 2.

Die Methoden unterscheiden sich darin, dass bei der KT eine Annährung in den Facettengelenken während der nach chirurgisch behandelten Patienten mit singulären thorakolumbalen 


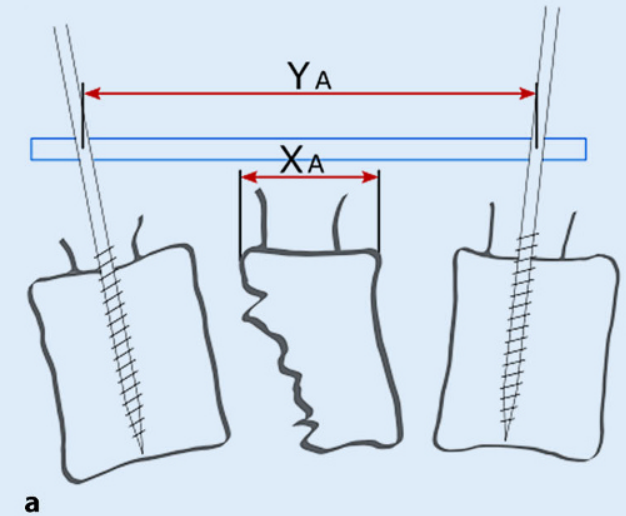

a

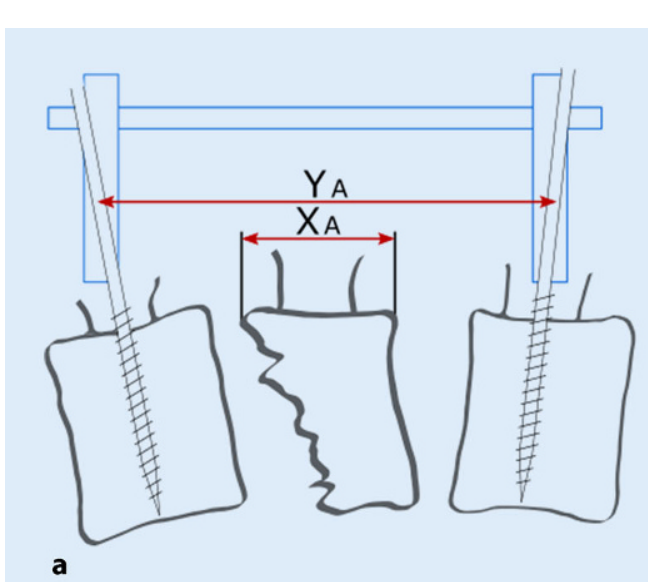

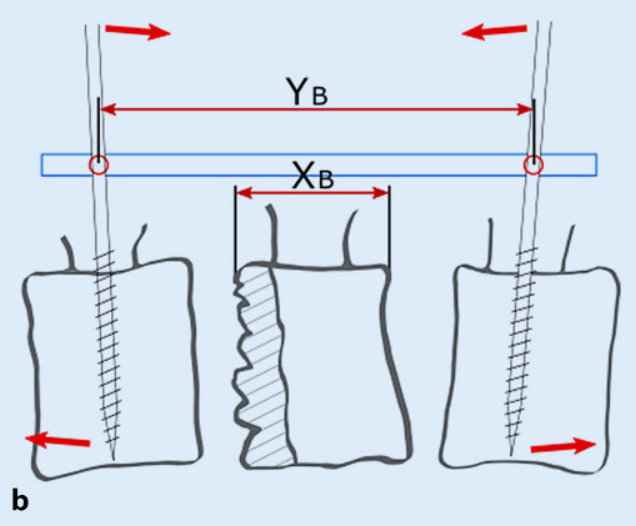

Abb. $1<$ Schematische Darstellung der AO-Schanz-Pin-Technik. a Situation zu Beginn, b Situation nach Repositionsmanöver; die Strecken $Y_{A}$ und $Y_{B}$ sind gleich lang Die Strecke $X_{B}$ ist deshalb länger als $X_{A}$. Es wird eine Distraktion der Hinterkante erreicht

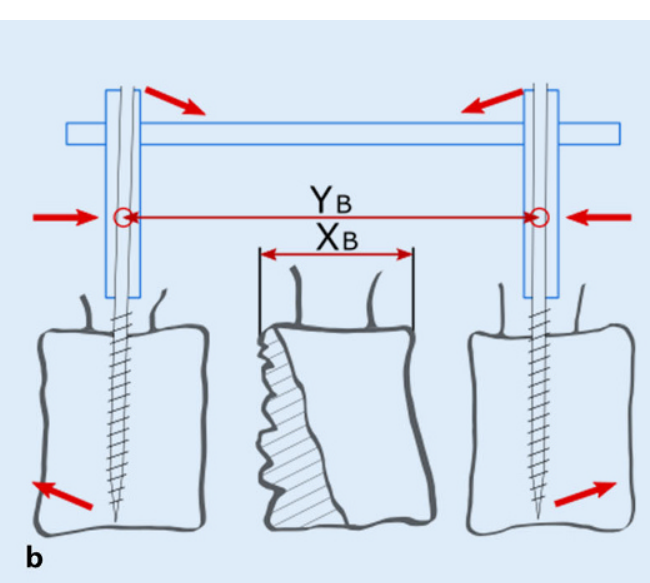

Abb. $2 \triangleleft$ Darstellung der Kluger-Technik. a Situation zu Beginn, b Situation nach Repositionsmanöver; die Strecke $Y_{B}$ ist kürzer als $Y_{A}$. Die Strecke $X_{B}$ ist deshalb genauso lang wie $X_{A}$. Es wird durch die Annährung der Rotationspunkte (roter Kreis) eine konstante Länge der Hinterkante gehalten. Eine Distraktion kann in einem 2. Schritt erfolgen

Lordosierung möglich wird, welche bei der AT wegen der Längenfixation gegen den Stab vor der Lordosierung nicht möglich ist.

Die HU wurden in der Technik nach Schreiber erhoben [7]. Hierzu wurden die HU aus jeweils 3 Ellipsen in axialen Schichten (kranial, mittig und kaudal) eines an den frakturierten Wirbelkörper angrenzenden Wirbelkörpers gemittelt. Die Flächen der Ellipsen wurden maximal gewählt, ohne dabei kortikale Anteile zu enthalten. Ein Schwellwert von $110 \mathrm{HU}$ wurde als Grenze zur Osteoporose entsprechend den Untersuchungen von Pickhardt et al. an 1867 CT- und DEXA-Datensätzen festgelegt [8].

Die Einteilung der Frakturschwere erfolgte anhand der AOSpine-Klassifikation [4]. Aufgrund der untersuchten lordosierenden Charakteristik beider Operationstechniken wurden Patienten mit B3Frakturen in der Analyse nicht berück- sichtigt, da hier i.d. R. einekyphosierende Korrektur erforderlich ist.

Der bGDW wurde in Röntgen- und CT-Aufnahmen zu den Zeitpunkten präoperativ, postoperativ und zur Nachuntersuchung (einen bis 3 Monate) gemessen. Negative Werte zeigen eine kyphotische und positive Werte eine lordotische Stellung des bGDW an.

\section{Normwerte für bGDW}

Auf der Grundlage der von Roussouly et al. [9] und Barrey et al. [10] erhobenen Werte für die lumbale Lordose (LL) und den „upper“ und „lower arc“ der LL wurden Normwerte für den bGDW abgeleitet, welche in • Tab. 1 dargestellt sind. Für eine Beschreibung der traumatisch bedingten Fehlstellung wurde die Differenz zwischen gemessenem bGDW zum präoperativen Zeitpunkt mit diesen Werten gebildet.

\section{Statistische Verfahren}

Intervallskalierte Daten wurden mittels Kolmogorov-Smirnov-Tests auf Normalverteilung überprüft. Unterschiede zu separaten Zeitpunkten zwischen den beiden Operationstechniken wurden verteilungsadäquat mit dem Mann-Whitney-U-Test oder T-Test für unabhängige Stichproben untersucht. Der Vergleich der bGDW mit den Normwerten erfolgte mittels verteilungsadäquaten Tests für abhängige Stichproben ( $t$-Test oder Wilcoxon-Test) separat für beide Operationstechniken.

Mittels Chi-Quadrat-Test wurden folgende Inhalte geprüft: Verteilung der AOSpine in den Gruppen AT und KT, Unterschiede im Erreichen der Normwerte zwischen den Gruppen AT und KT sowie Unterschiede im Anteil von Patienten mit weniger als $110 \mathrm{HU}$ in den Gruppen AT und KT. 
Unfallchirurg 2022 · 125:295-304 https://doi.org/10.1007/s00113-021-01013-7

(c) Der/die Autor(en) 2021

\section{B. W. Ullrich · M. Ottich · A. Lawson McLean · T. Mendel · G. O. Hofmann · P. Schenk}

\section{Lokales Wirbelsäulenprofil nach operativer Behandlung thorakolumbaler und lumbaler Frakturen. Einfluss von Repositionstechnik und Knochenqualität}

\section{Zusammenfassung}

Hintergrund. Ziel der Operation von Wirbelsäulenverletzungen ist eine stabile Ausheilung in physiologischer Stellung. Für offene und perkutane Operationen stehen unterschiedliche Techniken zur Verfügung. Fragestellung. Das Ausmaß der offenen Reposition und das Retentionspotenzial der Techniken nach AOSpine (AT) und nach Kluger (KT) sollen verglichen werden. Der Einfluss von Frakturmorphologie, Alter, Geschlecht und Knochenqualität auf Reposition und Retention werden untersucht.

Material und Methoden. In dieser monozentrischen retrospektiven Kohortenstudie wurden Daten von Patienten mit traumatischen thorakolumbalen und lumbalen Frakturen untersucht, welche entweder mit AT oder KT reponiert wurden. Mittels bisegmentalen Grund-Deckplatten-Winkels (bGDW) wurde die Stellung des verletzten Wirbelsäu- lenabschnitts beschrieben. Normalwerte für die bGDW wurden anhand von Literaturdaten angenommen. Die Veränderung des bGDW im zeitlichen Verlauf wurde unter Einbeziehung der Knochenqualität in Hounsfield Units (HU), der Verletzungsschwere nach AOSpine und des Patientenalters und -geschlechts analysiert.

Ergebnisse. Es wurden 151 Datensätze ausgewertet. Beide Methoden reponieren vom Umfang nicht unterschiedlich (AT $\left.10 \pm 6^{\circ}, \mathrm{KT} 11 \pm 8^{\circ} ; p=0,786\right)$. Im Followup trat ein Korrekturverlust von $-5 \pm 4^{\circ}$ auf. Die Technik $(p=0,998)$ hatte keinen Einfluss darauf. Die Frakturmorphologie zeigte einen knapp signifikanten Einfluss $(p=0,043)$. Niedrige HU korrelierten mit geringerem Repositionsumfang $(r=0,241, p<0,003)$ und größerem Korrekturverlust $(r=0,272$, $p<0,001)$ signifikant, aber schwach. In der Altersgruppe 50 bis 65 Jahre wiesen $21 \%$ der Männer und $43 \%$ der Frauen eine Knochenqualität von $\mathrm{HU}<110$ auf. Alter und HU korrelieren signifikant $(r=-0,701$, $p<0,001$ ).

Diskussion. Die Techniken sind gleichwertig bezüglich der Repositions- und Retentionseigenschaften. Der hohe Anteil von Patienten mit $\mathrm{HU}<110$ in der Gruppe unter 65 Jahren bei Frauen und Männern und der Einfluss auf Reposition und Retention weisen auf die Notwendigkeit einer präoperativen Knochendichtemessung hin.

\section{Schlüsselwörter}

Hounsfield Unit · Repositionstechnik · Posttraumatische Kyphose - Repositionsverlust . Knochenqualität

\section{Local spinal profile following operative treatment of thoracolumbar and lumbar fractures. Impact of reduction technique and bone quality}

\section{Abstract}

Background. The goal of surgery for spinal injuries is fracture reduction, fixation and stable healing in a physiological position. Several open and minimally invasive surgical techniques are available.

Objective. The extent of open reduction and the fixation potential achieved by the AOSpine (AT) and Kluger (KT) techniques were compared. The influence of fracture morphology, age, sex, and bone quality on fracture reduction and secure fixation was investigated.

Material and methods. In this monocentric retrospective cohort study data of patients with traumatic thoracolumbar and lumbar fractures treated by AT or KT were analyzed. The bisegmental kyphotic angle (bGDW) of each injured spinal segment was determined. Normal bGDW values were extrapolated from the literature. The change of bGDW over time was analyzed under consideration of the bone quality in Hounsfield units (HU), injury severity according to the AOSpine classification, gender and age of patients.

Results. A total of 151 data sets were evaluated. The AT and KT methods achieved a similar extent of reduction (AT $10 \pm 6^{\circ}, \mathrm{KT} 11 \pm 8^{\circ}$; $p=0.786$ ). In follow-up a mean reduction loss of $-5 \pm 4^{\circ}$ was seen. The technique had no influence on this $(p=0.998)$. The fracture morphology just managed to achieve a significant influence $(p=0.043)$. Low HU correlated significantly but weakly with lower extent of reduction $(r=0.241, p<0.003)$ and greater reduction loss $(r=0.272, p<0.001)$. In the age group $50-65$ years $21 \%$ of men and $43 \%$ of women had bone quality of $<110 \mathrm{HU}$. Age and $\mathrm{HU}$ were significantly correlated $(r=-0.701, p<0.001)$.

Conclusion. The AT and KT are equivalent in terms of reduction and secure fixation properties. The high proportion of male and female patients with $\mathrm{HU}<110$ in the age group under 65 years and the influence on reduction and secure fixation emphasize the need for preoperative bone densitometry.

Keywords

Hounsfield Unit · Posttraumatic kyphosis . Reduction technique - Reduction loss - Bone quality
Der Einfluss der Operationstechnik auf die Änderung des bGDW wurde mithilfe separater allgemeiner lineare Modelle für Messwiederholungen (rmANCOVA (Zeitpunkte $(2) \times$ Operationstechnik (2))) untersucht. Aufgrund des $p$-Wertes und eines möglichen Einflusses der AOSpine-Klassifikationen auf das Repositionsergebnis zwischen in den
Operationsgruppen (AT und KT) wurde diese als Kovariate verwendet.

Der Einfluss der HU auf den Umfang der Reposition und auf den Umfang der Änderung des bGDW im postoperativen Verlauf wurde mittels Pearson-Korrelation untersucht. Die Spearman-Korrelation wurde verwendet, um den Zusammenhang zwischen Frakturmorphologie nach AOSpine und dem Umfang der Reposition und der postoperativen Änderung des bGDW zu untersuchen.

Der Zusammenhang zwischen Alter und Knochenqualität wurde mittels Pearson-Korrelation untersucht. Unterschiede in der Knochenqualität zwischen den Geschlechtern wurde zum einen mittels $t$-Test für unabhängige Stichproben un- 
Tab. 1 Normwerte des bisegmentalen

Grund-Deckplatten-Winkels. (Basierend auf

den Daten von Roussouly et al. [9] und Barrey

et al. [10])

Bisegmen- Frakturier- Bisegmentale

tales Seg- ter Wirbel Normwerte

ment

\begin{tabular}{lll}
\hline Th9/Th11 & Th10 & $-5^{\circ}$ \\
\hline Th10/Th12 & Th11 & $-2^{\circ}$ \\
\hline Th11/L1 & Th12 & $2^{\circ}$ \\
\hline Th12/L2 & L1 & $7^{\circ}$ \\
L1/L3 & L2 & $10^{\circ}$ \\
L2/L4 & L3 & $15^{\circ}$ \\
L3/L5 & L4 & $25^{\circ}$ \\
L4/SWK 1 & L5 & $40^{\circ}$
\end{tabular}

tersucht und zum anderen altersadjustiert mittels einer univariaten Varianzanalyse.

Für die statistischen Analysen wurde SPSS V26 (IBM Corp. IBM SPSS Statistics for Windows; Fa. IBM Corp, Armonk, $\mathrm{NY}$ ) verwendet.

\section{Ergebnisse}

Es wurden Daten von 1538 Patienten gesichtet. Insgesamt konnten vollständige Datensätze von 151 Patienten (109 Männer, 42 Frauen) analysiert werden. Das Alter der Patienten lag im Mittel bei $45 \pm 16$ Jahren und unterschied sich nicht zwischen beiden Operationsgruppen $(p=0,415)$. Das Durchschnittsalter der Frauen (48 \pm 16 Jahre) lag im Mittel nur 3 Jahre über dem der Männer ( $45 \pm 16$ Jahre, $p=0,328$ ). Der Zeitraum der Nachuntersuchung betrug im Median 50 Tage $(p=0,069)$. Entsprechend der Operationstechnik wurden $102 \mathrm{~Pa}$ tienten der Gruppe AT (77\%) und 49 der Gruppe KT (23\%) zugeordnet. Die Verletzungshöhen und deren Verteilung auf die Gruppen AT und KT sind in - Tab. 2 dargestellt. Unterschiede in der Verteilung der Häufigkeit der frakturierten Wirbel zwischen beiden Gruppen bestanden nicht $(p=0,465)$. Frakturen von Th12, L1 und L2 machten $82 \%$ aller Verletzungen aus.

Die Häufigkeiten der Frakturmorphologie nach AOSpine-Klassifikation sind in 0 Tab. 3 dargestellt. Es waren Frakturen von $\mathrm{A} 1-\mathrm{C}$ vertreten; Patienten mit
Tab. 2 Darstellung der Häufigkeiten und deren prozentualen Verteilungen innerhalb der Operationsgruppen (AO-Schanz-Pin-

Technik [AT], Kluger-Technik [KT]) für die verletzten Wirbelkörper

\begin{tabular}{|c|c|c|c|c|}
\hline & & $\begin{array}{l}\text { AT, } n \\
(\%)\end{array}$ & $\begin{array}{l}\text { KT, } n \\
(\%)\end{array}$ & $\begin{array}{l}\text { Gesamt, } n \\
\text { (\%) }\end{array}$ \\
\hline \multirow{8}{*}{$\begin{array}{l}\text { Wir- } \\
\text { bel }\end{array}$} & Th10 & $1(1)$ & $1(2)$ & $2(1)$ \\
\hline & Th11 & $3(3)$ & $1(2)$ & $4(3)$ \\
\hline & Th12 & $20(20)$ & $13(27)$ & $33(22)$ \\
\hline & L1 & $45(44)$ & $22(45)$ & $67(44)$ \\
\hline & L2 & $14(14)$ & 7 (14) & $21(14)$ \\
\hline & L3 & $9(9)$ & - & $9(6)$ \\
\hline & L4 & $7(7)$ & $4(6)$ & $10(7)$ \\
\hline & L5 & $3(3)$ & $2(4)$ & $5(3)$ \\
\hline \multicolumn{2}{|c|}{ Gesamt } & 102 & 49 & 151 \\
\hline
\end{tabular}

B3 Frakturen wurden ausgeschlossen. A4-Frakturen traten in beiden Gruppen am häufigsten auf (AT: 49\%, KT: 74\%). Mit $22 \%$ in der Gruppe AT und $17 \%$ der Gruppe KT waren A3-Frakturen am zweithäufigsten. In Summe verteilen sich $74 \%$ der Frakturen auf diese beiden Frakturtypen. Im Mittel zeigten die Patienten der Gruppe AT knapp keine unterschiedliche Verteilung der Frakturtypen nach AOSpine als die der KT-Gruppe $(p=0,058)$.

\section{Frakturbedingte Deformität}

Verglichen mit den Normwerten zeigte sich präoperativ in beiden Gruppen eine signifikante frakturbedingte kyphotische Fehlstellung (AT: $-15 \pm 10^{\circ}, p<0,001$; KT: $\left.-15 \pm 10^{\circ}, p<0,001\right)$, welche sich zwischen den Operationsgruppen nicht sign. unterschied ( $p=0,837$, \Abb. 3a). Die Nullinie stellt hierbei den angenommenen Normwert höhenabhängig als Referenz dar. Die gemessenen präoperativen bGDW der Patienten unterschieden sich ebenfalls nicht zwischen beiden Operationsverfahren (AT: $-6 \pm 16^{\circ}$, KT: $-6 \pm 18^{\circ}, p=0,798,-$ Abb. 3b).

\section{Repositionsergebnis}

Durch die Operation wird eine Änderung der bGDW in der Gesamtpopulation von $11 \pm 8(p<0,001)$ herbeigeführt. Mit der AT wurde eine Reposition um $10 \pm 6$ und mit der KT um $11 \pm 8$ erzielt. Beide Operationstechniken unterscheiden sich
Tab. 3 Darstellung der Häufigkeiten und der Anteile der Frakturtypen nach AOSpine in den Operationsgruppen: AO-Schanz-PinTechnik (AT) und Kluger-Technik (KT)

\begin{tabular}{|c|c|c|c|c|}
\hline & & $\begin{array}{l}\text { AT, } n \\
(\%)\end{array}$ & $\begin{array}{l}\mathrm{KT}, n \\
(\%)\end{array}$ & $\begin{array}{l}\text { Gesamt, } n \\
\text { (\%) }\end{array}$ \\
\hline \multirow[t]{7}{*}{ AOSpine } & A1 & $4(4)$ & - & $4(3)$ \\
\hline & $A 2$ & $7(7)$ & $1(2)$ & $8(5)$ \\
\hline & A3 & $22(22)$ & $4(8)$ & $26(17)$ \\
\hline & A4 & $50(49)$ & $36(74)$ & $86(57)$ \\
\hline & B1 & $9(9)$ & $3(6)$ & $12(8)$ \\
\hline & B2 & $3(3)$ & $2(4)$ & $5(3)$ \\
\hline & C & $7(7)$ & $3(6)$ & $10(7)$ \\
\hline Gesamt & & 102 & 49 & 151 \\
\hline
\end{tabular}

nicht signifikant im Umfang der erzielten Reposition $(p=0,786)$. Der Interaktionseffekt zwischen Zeitpunkt und Operationstechnik war ebenfalls nicht signifikant $(p=0,476)$. Die Frakturschwere nach AOSpine als Kovariate zeigt keinen signifikanten Einfluss $(p=0,225)$ auf den Umfang der Reposition.

Bei 40 (26\%) Patienten (AT: $24 \%$, KT: $33 \%, p=0,244)$ wurden die Normwerte der bGDW durch die Reposition erreicht. Bei allen anderen Patienten (76\%) verblieb postoperativ ein kyphotischer Fehlstellungswinkel im Vergleich zu den Normwerten. Im Mittel unterschieden sich die postoperativen bGDW beider Gruppen signifikant von den Normwerten (AT $-5 \pm 8^{\circ}, p<0,001$; KT $-4 \pm 8^{\circ}$, $p<0,001)$.

\section{Postoperativer Verlauf}

Der bGDW verringerte sich im postoperativen Verlauf um $-5 \pm 4$ signifikant $(p<0,001)$. Die Operationstechnik $(p=0,998)$ zeigt sich nicht signifikant, die Verletzungsschwere $(p=0,043)$ hingegen zeigt einen knapp signifikanten Einfluss. Zur Nachuntersuchung betrugen die mittleren bGDW für AT $=-1 \pm 15^{\circ}$ und für $\mathrm{KT}=-1 \pm 16^{\circ} \quad(p=0,930)$. Verglichen mit den präoperativen bGDW verbleibt ein mittleres Repositionsergebnis von $5 \pm 8 \quad(p=0,002)$. Beide Operationstechniken unterscheiden sich nicht im verbliebenen Repositionsergebnis $(p=0,290)$. Ein Zusammenhang zwischen Umfang der ursprünglichen 

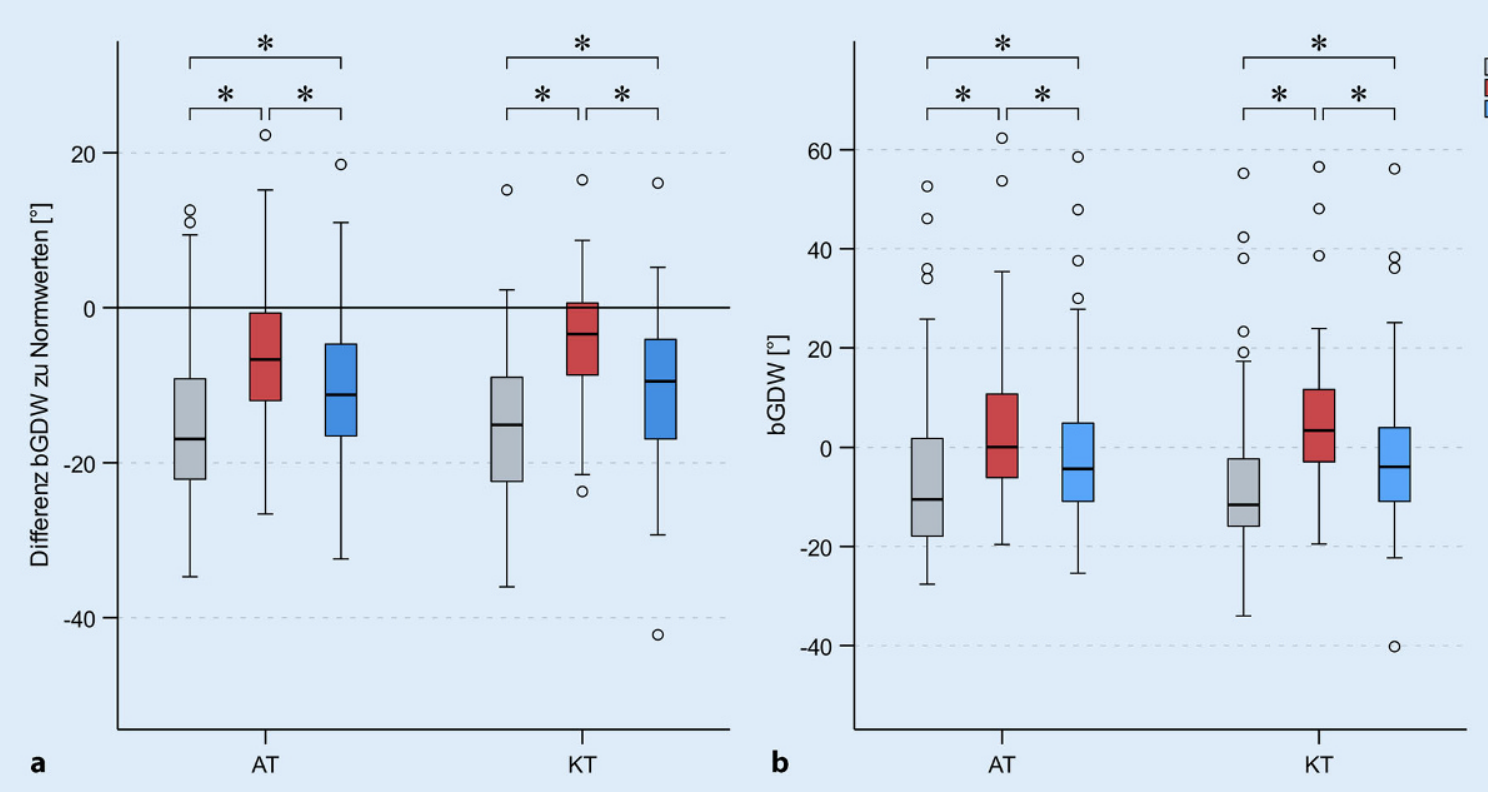

Abb. $3 \Delta$ a Darstellung der Abweichung der $b G D W$ zu den angenommenen Normwerten nach Roussouly et al. [9] und Barrey et al. [10] (höhenabhängig abgebildet, mit der Nulllinie als Referenz), welche für die beiden Operationsgruppen (AO-SchanzPin-Technik [AT] und Kluger-Technik [KT]) zu den 3 Untersuchungszeitpunkten dargestelltsind. $\mathbf{b}$ Darstellung der bGDWbeider Operationsgruppen ( $A T$ und $K T$ ) zu den 3 Untersuchungszeitpunkten. Signifikante Unterschiede zwischen den Zeitpunkten sind mit einem Asteriskus versehen. Die Fehlerbalken entsprechen den 1,5-fachen Interquartilsabstand; Kreise stellen Werte außerhalb dar

Reposition und dem Umfang des Repositionsverlusts lässt sich für beide Operationstechniken (AT $p=0,912$, KT $p=0,420)$ und über die komplette Stichprobe $(p=0,430)$ nicht zeigen.

Zum Zeitpunkt der Nachuntersuchung weisen nur noch $22(16 \%) \mathrm{Pa}$ tienten (AT: $16 \%$, KT: $12 \%, p=0,632$ ) einen bGDW oberhalb der Normwerte auf.

\section{Hounsfield Units}

Die HU-Werte lagen zwischen 45 und 318 (168 $\pm 58 \mathrm{HU})$. Unterschiede zwischen beiden Operationsgruppen konnten nicht festgestellt werden $(p=0,714)$. Bei insgesamt 28 Patienten (19\%) wurden HU-Werte kleiner als 110 gemessen. Der Anteil an Patienten mit HU kleiner als 110 war in beiden Operationsgruppen vergleichbar (AT: $20 \%$, KT: $16 \%$, $p=0,823)$. Der Zusammenhang zwischen der Knochenqualität und dem Umfang der Reposition ist signifikant $(\mathrm{r}=0,241, p=0,003)$. Dies bedeutet, dass bei Patienten mit höheren HU eine umfangreichere Reposition erzielt wurde. Mit einem $r^{2}$ von 0,06 ist dieser $\mathrm{Zu}$ - sammenhang jedoch als sehr gering zu bezeichnen ( Abb. 4a). Auf den Repositionsverlust im Nachuntersuchungszeitraum zeigen die HU-Werte ebenfalls einen signifikanten Einfluss $(r=0,272$, $p=0,001$ ). Niedrigere HU sind demnach mit einem größeren Repositionsverlust verbunden $(\bullet \mathbf{A b b} . \mathbf{4 b})$. Auch hier ist der Zusammenhang als gering zu bezeichnen $\left(r^{2}=0,07\right)$.

\section{AOSpine-Klassifikation}

Ein Zusammenhang zwischen der Frakturmorphologie und der erzielten Reposition besteht schwach, aber signifikant $(r=0,189, p=0,020)$ in dem Sinne, dass bei einer höhergradigen Fraktur auch ein größerer Repositionsumfang erzielt wird. Für den Korrekturverlust findet sich dieser Zusammenhang nicht $(p=0,457)$. Der Zusammenhang zwischen Knochenqualität und Frakturmorphologie zeigt sich als sehr schwach $(\mathrm{r}=0,186)$, aber signifikant $(p=0,022)$. Patienten mit hohen HU-Werten zeigen eine Tendenz zu höhergradigeren Verletzungen nach AOSpine (• Abb. 5).

\section{Beziehung HU, Alter, Geschlecht}

Insgesamt 132 Patienten (87\%) waren jünger als 65 Jahre. Hiervon wiesen 17 (13\%) eine schlechte Knochenqualität $(\mathrm{HU}<110)$ auf. Männer ( $n=11$ von 96) und Frauen ( $n=6$ von 36$)$ verteilten sich somit $\mathrm{zu} 11$ und $17 \%$.

Insgesamt 43 aller Patienten waren zwischen 50 und 65 Jahre alt. Hiervon wiesen $12(43 \%)$ (6 von 29 Männern (21\%) und 6 von 14 Frauen $(43 \%))$ eine schlechte Knochenqualität $(\mathrm{HU}<110)$ auf.

Mit zunehmendem Alter zeigt sich eine Verminderung der Knochenqualität $(\mathrm{r}=-0,701, p<0,001)$. Der Zusammenhang ist als stark zu bezeichnen und in - Abb. 6 dargestellt. Die geschlechtsspezifische Betrachtung der Knochenqualität zeigt im Mittel keine Unterschiede zwischen Männern $(170 \pm 58 \mathrm{HU})$ und Frauen $(163 \pm 59 \mathrm{HU}, p=0,518)$. Unter Berücksichtigung des Alters als signifikante Kovariate $(p<0,001)$ zeigt sich ebenfalls kein signifikanter Unterschied $(p=0,937)$. Die altersadjustierten Mittelwerte der HU über die komplette Stichprobe liegen für Männern bei 168 

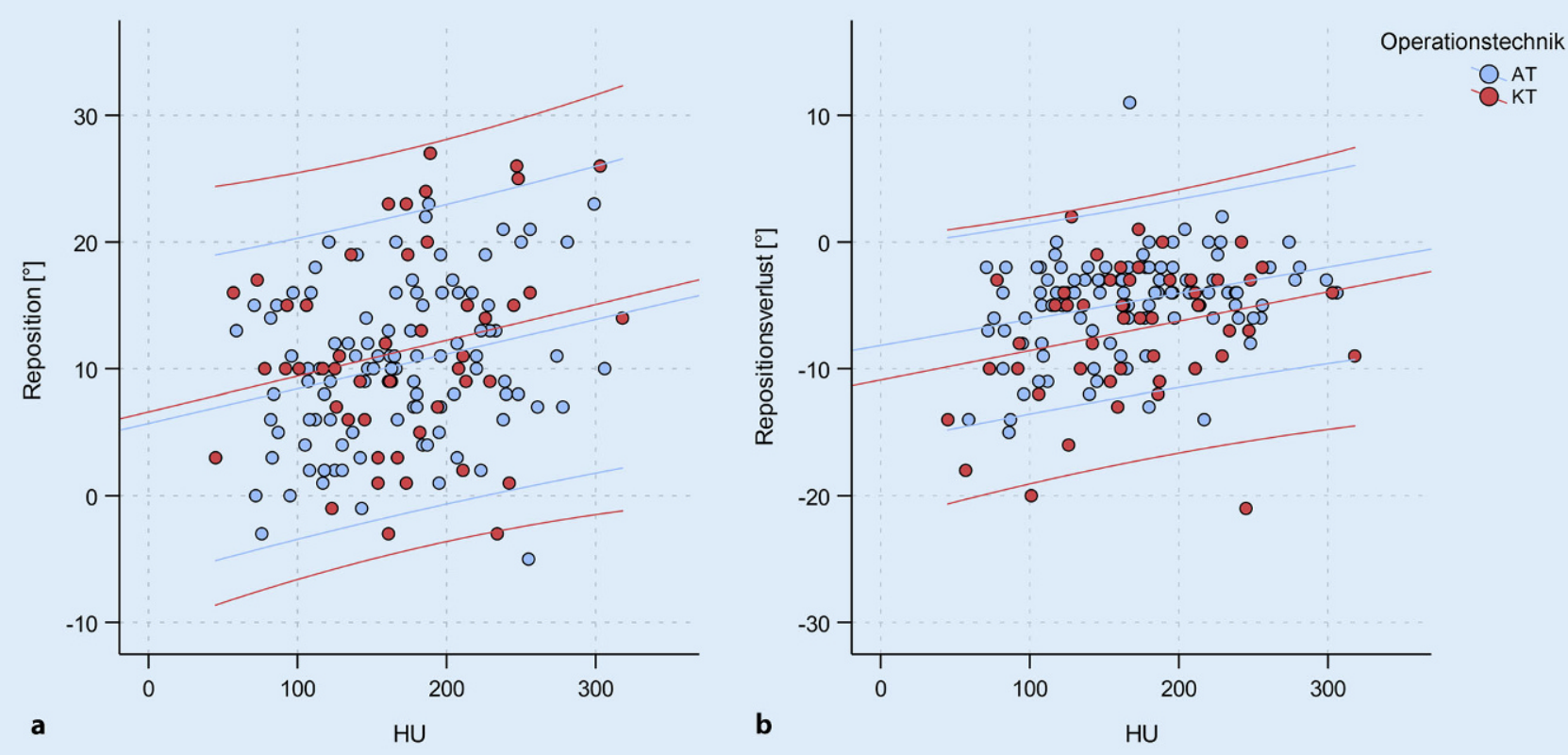

Abb. $4 \Delta$ Darstellung des Zusammenhangs der Knochenqualität, ermittelt in $H U$, und des Umfangs der Reposition (a) und des Umfangs des Repositionsverlustes (b). Die Operationsgruppen sind farblich gekennzeichnet (AO-Schanz-Pin-Technik [AT] hellblau, Kluger-Technik [KT] rot). Die jeweiligen Regressionsgeraden und dazugehörigen Konfidenzintervalle (95\%) sind als farblich korrespondierende Linien eingezeichnet

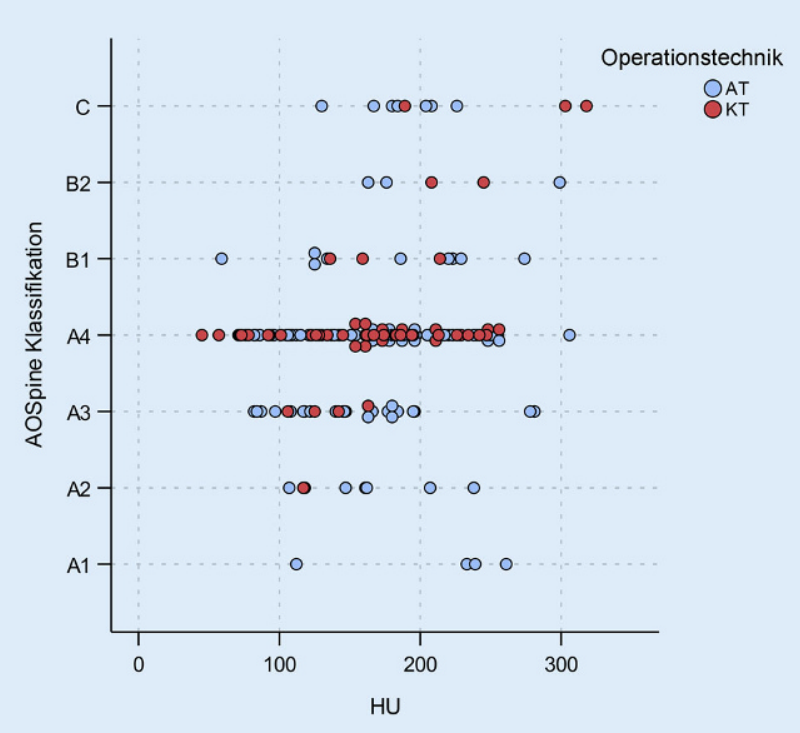

Abb. 5 \& Darstellung der Knochenqualität anhand von HU-Werten und der Frakturklassifikation anhand AOSpine der Patienten beider Operationsgruppen (AO-Schanz-Pin-Technik [AT], Kluger-Technik [KT])

(95. Konfidenzintervall 160-176) und für Frauen bei 168 (95. Konfidenzintervall 156-181).

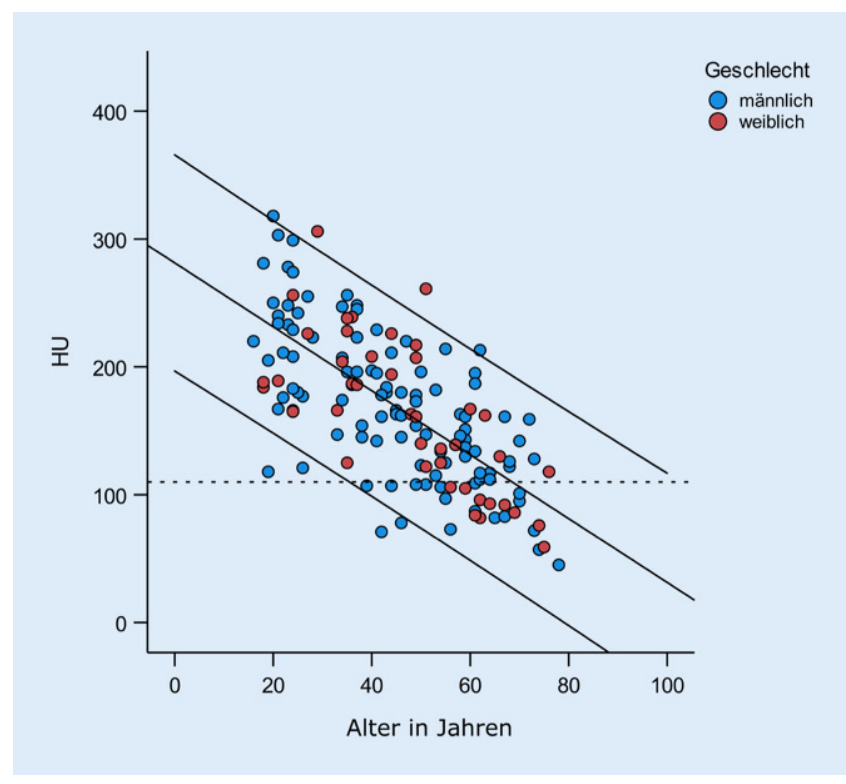

Abb. $6 \Delta$ Darstellung der HU-Werte in Abhängigkeit vom Alter. Der Zusammenhang und die zugehörigen 95\%-Konfidenzintervalle sind als durchgezogene schwarze Line eingezeichnet. Die gestrichelte Linie ist bei $110 \mathrm{HU}$. Das Geschlecht der Patienten ist farblich gekennzeichnet (Männer blau, Frauen rot)

\section{Diskussion}

Auffällig ist, dass nur ca. $10 \%$ der durch die Suchanfrage ausgegebenen Datensätze für die Auswertung herangezogen werden konnten. Die häufigsten Grün- de für die Reduktion bei gleichzeitiger Homogenisierung der Stichprobe waren: fehlende Verlaufsdokumentationen, unvollständige Röntgendokumentation, multisegmentale Stabilisierung, polyaxiale oder minimal-invasive Fixateur- 
Systeme. Da keine systematische Selektion stattgefunden hat, ist davon auszugehen, dass die Ergebnisse trotzdem repräsentativ sind. Primär engere Auswahlkriterien hätten eine präzisere Ausgabe ermöglicht. Die Übertragbarkeit und Generalisierung unserer Ergebnisse auf die ursprüngliche Kohorte ist somit aufgrund des geschrumpften Stichprobenumfangs eingeschränkt.

\section{Repositionstechniken}

Durch die offene Reposition ist eine relevante Korrektur der unfallbedingten Fehlstellung möglich, wie auch in der zweiten internetbasierten Multizenterstudie der Deutschen Gesellschaft für Unfallchirurgie (MCS ll) gezeigt werden konnte [11]. Das Repositionsausmaß von 10 können wir bestätigen. Die Daten der MSC 11 zeigen, dass das initiale Repositionsergebnis insbesondere ohne zusätzliche ventrale Stabilisierung nicht gehalten werden konnte [12]. Dass ein erheblicher Repositionsverlust im Zeitraum vor einer ventralen Stabilisierung eintritt, können wir mit dieser Untersuchung bestätigen. Daten aus der hier dargestellten Kohorte nach ventraler Abstützung durch einen "Stand-alone“Wirbelkörperersatz (VLIFT ${ }^{\circledR}$ Stryker, Kalamazoo, MI, USA) zeigten aber auch, dass die Sinterung nach einer ventralen Stabilisierung von der Knochenqualität abhängig ist. Je schlechter die Knochenqualität, desto größer die Sinterung. Ausschließlich in der Gruppe mit HUWerten > 180 konnte eine minimale Sinterung detektiert werden [13].

\section{Korrekturverlust}

Der Korrekturverlust von im Mittel - 5 bei alleiniger dorsaler Instrumentierung ist signifikant und stützt die Ergebnisse der MSC $11\left(-7^{\circ}\right)$ und der Arbeit von Spiegl et al. ( -5 nach 60 Tagen) [14]. Aussagen zurklinischen Bedeutung dieser Fehlstellung können nicht getroffen werden, da im Rahmen dieser Untersuchung keine strukturierte Erfassung klinischer Parameter erfolgte. In einer monozentrischen prospektiven Pilotstudie, bei der die Indikation zur anterioren Fusion randomisiert gestellt wurde, bestätigte sich ein signifikant geringerer Repositionsverlust und zeigten sich tendenziell klinisch bessere Ergebnisse im ODI für die dorsoventral stabilisierten Patienten [15]. Der Wert der additiven ventralen Stabilisierung wird weiterhin kritisch diskutiert, wie in einem Review von Tan et al. aus 2020 zum Ausdruck kommt, welches allerdings die MSC-Daten $[11,12,16]$ nicht berücksichtigt [17].

\section{Knochenqualität}

Die Knochenqualität wurde in der MSCll nicht betrachtet. HU aus opportunistischen CT-Scans können als Surrogatparameter für die Knochenqualität herangezogen werden $[7,8]$. Pickhardt et al. [8] belegten, dass Werte $<110 \mathrm{HU}$ eine mehr als $90 \%$ ige Spezifität für die Vorhersage einer Osteoporose haben. Legt man dies zugrunde, zeigen die hier vorgestellten Daten, dass ein relevanter Anteil der unter 65-jährigen Patienten (Frauen 17\%, Männer $11 \%$ ) sehr wahrscheinlich eine osteoporotische Knochenqualität aufwies. Unabhängig davon zeigen niedrige HU-Werte ein erhöhtes Risiko für Komplikationen wie Sinterungen von Cages [13], Lockerungen von Pedikelschrauben [18] und Anschlussfrakturen [19] an. Sie können somit dienlich für die Operationsplanung sein. Die hier erhobenen Daten zeigen einen signifikanten Einfluss der HU auf das Repositionsergebnis (je niedriger die HU, umso kleiner der Repositionsweg) und den Korrekturverlust (je niedriger die HU, umso größer der Repositionsverlust). Aufgrund der breiten Streuung der Werte sind die gefundenen Zusammenhänge als sehr gering zu bezeichnen, und die Aussagekraft der Ergebnisse ist limitiert.

Interessant an den Daten der MSC 11 ist, dass ab einem Patientenalter von 41 banale Stürze einen relevanten Anteil der Unfallursache darstellen. Dies deutet darauf hin, dass der Anteil an Patienten mit verminderter Knochenqualität in der MCS 11 auch bei Patienten zwischen 40 und 60 Jahren höher gewesen sein könnte als angenommen.

\section{Frakturbedingte Deformität}

Das Ziel der Reposition ist die Korrektur der Fehlstellung auf das ursprüngliche, individuelle Wirbelsäulenprofil. In der Regel existieren keine prätraumatischen Aufnahmen der Wirbelsäule. Es kommt hinzu, dass bei wirbelsäulenverletzten Patienten Untersuchungen der Wirbelsäule gemäß der „Leitlinie der Bundesärztekammer zur Qualitätssicherung in der Röntgendiagnostik“ [20] im Stehen, die üblicherweise zur Feststellung des individuellen sagittalen und koronaren Profils zur Anwendung kommen, nicht möglich sind. Wirbelsäulenverletzungen bedingen in der Regel eine kyphotische Deformität, die im Verlauf erheblich zunehmen kann [21], sodass eine Bestimmung des individuellen Profils, wie es vor der Verletzung bestanden hat, nicht möglich ist.

\section{Prätraumatisches lokales Wirbelsäulenprofil}

Nach Kenntnis der Autoren gibt es kein etabliertes Verfahren, mit welchem bei einem im Liegen untersuchten wirbelsäulenverletzten Patienten vorbestehende physiologische bGDW der verletzten Segmente abgeleitet werden können. Um dieses Problem zu adressieren, wurden für diese Arbeit, basierend auf den Daten von Roussouly et al. [9] und Barrey et al. [10], Normwerte angenommen. Kritisch daran ist, dass die starken interindividuellen Schwankungen mit diesen Mittelwerten nicht erfasst werden. Betrachtet man die Lordosetypen nach LeHuec und die damit einhergehende Varianz der Höhe des Inflexionspunktes [9] kann der physiologische Wert für den bGDW der Segmente Th12/L1/L2 zwischen -10 und $+10^{\circ}$ variieren.

Dass durch die Reposition die angenommenen Normwerte nur in einer Minderzahl der Fälle erreicht wurden, kann durch oben genannte Limitation bedingt sein oder tatsächlich durch einen unzureichenden Repositionsweg verursacht sein. Diese Frage kann mit den vorhandenen Daten nicht geprüft werden.

Seit 2013 steht als Ergebnis eines globalen Konsensusprozesses die AOSpineKlassifikation für die Einteilung der 
Schwere thorakolumbaler Verletzungen zur Verfügung [4], welche vorangegangene Klassifikationen wie die nach Magerl [22], McCormack [23] und die TLICS [24] aufgenommen und zusammengeführt hat. Somit scheint die AOSpineKlassifikation die Verletzungsschwere umfänglich zu beschreiben, sodass auf eine Darstellung der Ergebnisse im Bezug zu den anderen Klassifikationen (Magerl, McCormack und TLICS) nicht erfolgte.

Dass bei Patienten mit niedrigeren HU eher niedriggradigere Frakturen auftreten, kann mit den vorliegenden Daten nicht erklärt werden. Möglicherweise führen Niedrigenergietraumen bei Patienten mit schlechterer Knochenqualität häufiger zu einfacheren Frakturen als bei Knochengesunden. Informationen zum Unfallhergang mit einer Kategorisierung nach einwirkender Energie in Analogie zur MSC 11 wären geeignet, den Unfallhergang als Kovariate zu untersuchen.

\section{HU, Alter und Geschlecht}

Die Ergebnisse zeigen die bekannte negative Korrelation [25] zwischen Alter und HU. Interessant ist, dass sich altersbereinigt kein Unterschied zwischen Männern und Frauen finden lässt. Demnach scheint in unserer Kohorte das Alter einen stärkeren Einfluss auf die HU zu haben als das Geschlecht. Mögliche Ursache hierfür kann sein, dass das Vorhandensein einer Wirbelfraktur im $\mathrm{Zu}$ sammenhang mit der Knochenqualität steht und somit dieses Ergebnis auf einem Selektionsbias beruht.

\section{Fazit für die Praxis}

\begin{tabular}{|c|}
\hline $\begin{array}{l}\text { Wirbelfrakturen zu reponieren. } \\
\text { Der Anteil von Patienten mit erheb- } \\
\text { lich reduzierter Knochenqualität liec } \\
\text { bei unter 65-Jährigen in einem für d } \\
\text { Therapie relevanten Ausmaß. } \\
\text { Die Hounsfield Unit-Werte aus na- } \\
\text { tiven CT-Scans als Maß für die Kno- } \\
\text { chenqualität haben einen Einfluss } \\
\text { auf das Repositionsergebnis und }\end{array}$ \\
\hline
\end{tabular}

den Korrekturverlust und sollten präoperativ bestimmt werden.

- Bereits nach kurzer Zeit ist unabhängig von der Operationstechnik ein signifikanter Repositionsverlust nachweisbar.

\section{Korrespondenzadresse}

\section{Dr. Bernhard Wilhelm Ullrich}

Klinik für Unfall-, Hand- und Wiederherstellungschirurgie, Universitätsklinikum Jena

Am Klinikum 1, 07747 Jena, Deutschland b.w.ullrich@me.com

Funding. Open Access funding enabled and organized by Projekt DEAL.

\section{Einhaltung ethischer Richtlinien}

Interessenkonflikt. B.W. Ullrich, M. Ottich, A. Lawson McLean, T. Mendel, G.O. Hofmann und P. Schenk geben an, dass kein Interessenkonflikt besteht.

Für diesen Beitrag wurden von den Autoren keine Studien an Menschen oder Tieren durchgeführt. Für die aufgeführten Studien gelten die jeweils dort angegebenen ethischen Richtlinien. Diese retrospektive Studie erfolgte nach Konsultation der zuständigen Ethikkommission und im Einklang mit nationalem Recht.

Open Access. Dieser Artikel wird unter der Creative Commons Namensnennung 4.0 International Lizenz veröffentlicht, welche die Nutzung, Vervielfältigung, Bearbeitung, Verbreitung und Wiedergabe in jeglichem Medium und Format erlaubt, sofern Sie den/die ursprünglichen Autor(en) und die Quelle ordnungsgemäß nennen, einen Link zur Creative Commons Lizenz beifügen und angeben, ob Änderungen vorgenommen wurden.

Die in diesem Artikel enthaltenen Bilder und sonstiges Drittmaterial unterliegen ebenfalls der genannten Creative Commons Lizenz, sofern sich aus der Abbildungslegende nichts anderes ergibt. Sofern das betreffende Material nicht unter der genannten Creative Commons Lizenz steht und die betreffende Handlung nicht nach gesetzlichen Vorschriften erlaubt ist, ist für die oben aufgeführten Weiterverwendungen des $\mathrm{Ma}$ terials die Einwilligung des jeweiligen Rechteinhabers einzuholen.

Weitere Details zur Lizenz entnehmen Sie bitte der Lizenzinformation auf http://creativecommons.org/ licenses/by/4.0/deed.de.

\section{Literatur}

1. Kumar R, Lim J, Mekary RA, Rattani A, Dewan MC, Sharif SY et al (2018) Traumatic spinal injury: global epidemiology and worldwide volume. World Neurosurg 113:e345-e363. https://doi.org/ 10.1016/j.wneu.2018.02.033
2. (2020) https://surgeryreference.aofoundation. org/spine/trauma/thoracolumbar/a4/posteriorshort-segment-fixation-with-schanzpinszugegriffen.Zugegriffen:24. Aug. 2020

3. Kluger P, Gerner HJ (1986) Mechanical principle of external fixation for dorsal stabilization of thoracic and lumbar vertebrae. Unfallchirurgie 12(2):68-79

4. Vaccaro AR, Oner C, Kepler CK, Dvorak M, Schnake K, Bellabarba C et al (2013) AOSpine thoracolumbar spine injury classification system: fracture description, neurological status, and key modifiers. Spine 38(23):2028-2037

5. Verheyden AP, Holzl A, Ekkerlein H, Gercek E, Hauck S, Josten C et al (2011) Recommendations for the treatment of thoracolumbar and lumbar spine injuries. Unfallchirurg 114(1):9-16. https:// doi.org/10.1007/s00113-010-1934-1

6. Dick W, Kluger $P$, Magerl F, Woersdorfer $O$, Zach G (1985) A new device for internal fixation of thoracolumbar and lumbar spine fractures: the 'fixateur interne. Paraplegia 23(4):225-232. https://doi.org/10.1038/sc.1985.38

7. Schreiber JJ, Anderson PA, Rosas HG, Buchholz AL, Au AG (2011) Hounsfield units for assessing bone mineral density and strength: a tool for osteoporosis management. J Bone Joint Surg Am 93(11):1057-1063. https://doi.org/10.2106/JBJS. J.00160

8. Pickhardt PJ, Pooler BD, Lauder T, del Rio AM, Bruce RJ, Binkley N (2013) Opportunistic screening for osteoporosis using abdominal computed tomography scans obtained for other indications. Ann Intern Med 158(8):588-595. https://doi.org/ 10.7326/0003-4819-158-8-201304160-00003

9. Roussouly P, Gollogly S, Berthonnaud E, Dimnet J (2005) Classification of the normal variation in the sagittal alignment of the human lumbar spine and pelvis in the standing position. Spine 30(3):346-353

10. Barrey C, Roussouly P, Le Huec JC, D'Acunzi G, Perrin G (2013) Compensatory mechanisms contributing to keep the sagittal balance of the spine. Eur Spine J 22(Suppl 6):S834-S841. https:// doi.org/10.1007/s00586-013-3030-z

11. Reinhold M, KnopC, Beisse R, Audige L, Kandziora F, Pizanis A et al (2009) Operative treatment of traumatic fractures of the thorax and lumbar spine. Part Il:surgical treatment and radiological findings. Unfallchirurg 112(2):149-167. https://doi.org/10. 1007/s00113-008-1538-1

12. Reinhold M, KnopC, Beisse R, Audige L, Kandziora F, Pizanis A et al (2010) Operative treatment of 733 patients with acute thoracolumbar spinal injuries: comprehensive results from the second, prospective, Internet-based multicenter study of the Spine Study Group of the German Association of Trauma Surgery. Eur Spine J 19(10):1657-1676. https://doi.org/10.1007/s00586-010-1451-5

13. Ullrich BW, Schenk P, Spiegl UJ, Mendel T, Hofmann GO (2018) Hounsfield units as predictor for cage subsidence and loss of reduction: following posterior-anterior stabilization in thoracolumbar spine fractures. Eur Spine J. https://doi.org/10.1007/s00586-018-5792-9

14. Spiegl UJ, Jarvers JS, Heyde CE, Glasmacher S, Von der Hoh N, Josten C (2015) Delayed indications for additive ventral treatment of thoracolumbar burst fractures: What correction loss is to be expected. Unfallchirurg. https://doi.org/10.1007/ s00113-015-0056-1

15. Scholz M, Kandziora F, Tschauder T, Kremer M, Pingel A (2018) Prospective randomized controlled comparison of posterior vs. posterior-anterior stabilization of thoracolumbar incomplete 
cranial burst fractures in neurological intact patients: the RASPUTHINE pilot study. Eur Spine J 27(12):3016-3024

16. Reinhold M, KnopC, Beisse R, Audige L, Kandziora F, Pizanis A et al (2009) Operative treatment of traumatic fractures of the thoracic and lumbar spinal column: part III: follow up data]. Unfallchirurg 112(3):294-316. https://doi.org/10. 1007/s00113-008-1539-0

17. Tan T, Donohoe TJ, Huang MS-J, Rutges J, Marion T, Mathew J et al (2020) Does combined anteriorposterior approach improve outcomes compared with posterioronly approach in traumatic thoracolumbar burst fractures?: a systematic review. Asian Spine J 14(3):388

18. Bredow J, Boese CK, Werner CM, Siewe J, Lohrer L, Zarghooni K et al (2016) Predictive validity of preoperative $\mathrm{CT}$ scans and the risk of pedicle screw loosening in spinal surgery. Arch Orthop Trauma Surg. https://doi.org/10.1007/s00402-016-2487-

19. Meredith DS, Schreiber JJ, Taher F, Cammisa FP Jr., Girardi FP (2013) Lower preoperative Houns field unit measurements are associated with adjacent segment fracture after spinal fusion. Spine 38(5):415-418. https://doi.org/10.1097/ BRS.0b013e31826ff084

20. (2020) https://www.bundesaerztekammer.de/ fileadmin/user_upload/downloads/ LeitRoentgen2008Korr2.pdf. Zugegriffen: 24. Aug. 2020

21. Vaccaro AR, Silber JS (2001) Post-traumatic spinal deformity. Spine 26(24S):S111-S8

22. Magerl F, Aebi M, Gertzbein SD, Harms J, Nazarian S (1994) A comprehensive classification of thoracic and lumbar injuries. Eur Spine J3(4):184-201

23. McCormack T, Karaikovic E, Gaines RW (1994) The load sharing classification of spine fractures. Spine 19(15):1741-1744

24. Vaccaro AR, Lehman RA Jr, Hurlbert RJ, Anderson PA, Harris M, Hedlund R et al (2005) A new classification of thoracolumbar injuries: the importance of injury morphology, the integrity of the posterior ligamentous complex, and neurologic status. Spine 30(20):2325-2333

25. Lee S, Chung CK, Oh SH, Park SB (2013) Correlation between bone mineral density measured by dual-energy X-ray absorptiometry and Hounsfield units measured by diagnostic CT in lumbar spine. JKorean Neurosurg Soc 54(5):384-389

\section{Journal Club in Der Unfallchirurg - jetzt einreichen!}

Sie haben eine spannende Studie gelesen? Kommentieren Sie sie für Ihre Kolleg*innen!

\section{Liebe Leserinnen und Leser, liebe Autorinnen und Autoren,}

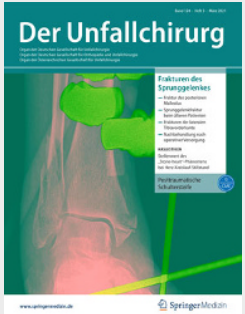

haben Sie kürzlich eine spannende Studie gelesen, die Sie interessiert und begeistert hat? Haben Sie Lust diese aufzubereiten und Ihren Kolleg*innen vorzustellen? Reichen Sie jetzt Ihr Manuskript für die beliebte Rubrik „Journal Club” ein!

Ergreifen Sie die Gelegenheit Der Unfallchirurg als Autor*in aktiv mitzugestalten und erleichtern Sie unseren Leser*innen die unkomplizierte Umsetzung neuester Forschungsergebnisse in ihrer täglichen Praxis.

Um Ihnen bei der Manuskripterstellung behilflich zu sein und unseren Leser*innen bei der Lektüre Ihres Beitrags den größtmöglichen Nutzen zu bieten, geben wir Ihnen gerne folgende Checkliste an die Hand:

- Die besprochene Studie sollte für die praktische Tätigkeit unserer Leser*innen hohe diagnostische oder therapeutische Relevanz haben und innerhalb der letzten 12 Monate publiziert worden sein.

- Gesamtumfang: ca. 6.000-9.000 Zeichen (inkl. Leerzeichen)

- Text bitte immer als offene Datei einreichen (.doc/.docx)

- Vollständige Anschrift des Korrespondenzautors mit E-Mail-Adresse sowie Portraitfoto

- Kurzer, prägnanter Beitragstitel, ggf. erläuternder Untertitel

- Vollständige Angabe der besprochenen Originalpublikation

- Kurze Zusammenfassung der Originalstudie

- Ihr Kommentar zur Studie: Studiendesign, Zusammenfassung der Ergebnisse, Stärken und Schwächen der Studie, Einschätzung des ableitbaren Patientenutzens und der Praxisrelevanz

- Max. 5 Literaturzitate

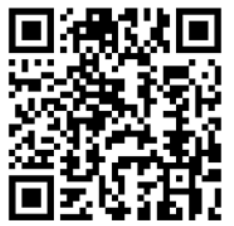

Ein Template für die Manuskripterstellung sowie einen Musterbeitrag finden Sle unter www.springer.com/journal/113/submissionguidelines oder nutzen Sie den nebenstehenden QR-Code.

\section{Reichen Sie Ihr Manuskript jetzt ein!}

Bitte kontaktieren Sie zur Einreichung von Manuskripten und bei Fragen zur Manuskriptgestaltung die Verlagsredaktion:

Anna Sittig

Tel.: 06221/4878304

anna.sittig@springer.com

Alle Beiträge in Der Unfallchirurg durchlaufen einen Peer-Review-Prozess.

Informieren Sie sich über alle Vorteile für unsere Autor*innen unter www.SpringerMedizin.de/Schreiben 
Hier steht eine Anzeige.

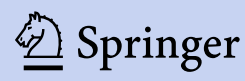

